\title{
FPGA-based Klystron Linearization Implementations in Scope of ILC
}

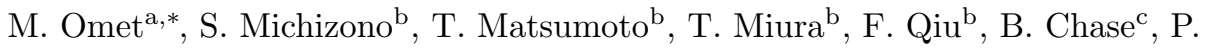 \\ Varghese $^{\text {c }}$, H. Schlarb ${ }^{\text {d }}$, J. Branlard ${ }^{\text {d }}$, W. Cichalewski ${ }^{\mathrm{e}}$ \\ ${ }^{a}$ The Graduate University for Advanced Studies, Hayama, Japan \\ ${ }^{b}$ The Graduate University for Advanced Studies / High Energy Accelerator Research \\ Organization, Tsukuba, Japan \\ ${ }^{c}$ Fermi National Accelerator Laboratory, Batavia, USA \\ ${ }^{d}$ Deutsches Elektronen-Synchrotron, Hamburg, Germany \\ ${ }^{e}$ Lodz University of Technology, Lodz, Poland
}

\begin{abstract}
We report the development and implementation of four FPGA-based predistortiontype klystron linearization algorithms. Klystron linearization is essential for the realization of ILC, since it is required to operate the klystrons $7 \%$ in power below their saturation. The work presented was performed in international collaborations at the Fermi National Accelerator Laboratory (FNAL), USA and the Deutsches Elektronen Synchrotron (DESY), Germany. With the new developed algorithms the generation of correction factors on the FPGA was improved compared to past algorithms, avoiding quantization and decreasing memory requirements. At FNAL three algorithms were tested at the Advanced Superconducting Test Accelerator (ASTA) demonstrating a successful implementation for one algorithm and a proof of principle for two algorithms. The functionality of the algorithm implemented at DESY was demonstrated successfully in a simulation.

Beside this a proof of principle of an FPGA-based klystron and cavity simulator implemented at the High Energy Accelerator Research Organization (KEK), Japan was demonstrated. Its purpose is to allow the development and test of digital LLRF control systems including klystron linearization algorithms
\end{abstract}

\footnotetext{
*momet@post.kek.jp
} 
in times no actual klystron and cavity are available.

Keywords: ILC, klystron linearization, FPGA, klystron-cavity simulator

\section{Introduction}

Klystrons are widely used for the high level radio frequency (HLRF) generation for driving cavities in particle accelerators. It is planned to adapt this technology at the International Linear Collider (ILC) [1,2. At ILC the klystrons 5 will be one element in the chain of the digital low level RF (LLRF) control loop. Typically the input to output characteristics of a klystron in both amplitude and phase are not linear. A schematic of a typical input-to-output amplitude characteristic is shown in Figure 1 in black. In order to perform field regulation, typically klystrons are operated about $40 \%$ below saturation. In order to operate the klystrons at ILC most cost effectively, it is intended to operate them $7 \%$ (in power) below the point of saturation. At this point the slope of the input-power-to-output-power characteristics is only about $\frac{1}{10}$ compared to the slope at the linear region. Since the control gain is proportional to this slope, it also degrades close to the point of saturation. In order to keep the feedback effective, it is required to keep the slope of the input-to-output amplitude characteristic constant. Furthermore it is preferable to introduce an output amplitude limiter and to eliminate any output phase rotation. The desired klystron output characteristics in amplitude and phase are represented in Figure 1 as red dashed lines in the respective plots. Such output characteristics can be accomplished by using a klystron linearization. The linearization algorithms described in the following are predistortion-type linearizations, implemented in the firmware of the Field Programmable Gate Array (FPGA), on which beside others the controller is located. The predistortion added is the inverse of the non-linear characteristics of the klystron. It is typically generated in dependency of the signal amplitude and applied after the controller and the addition of feedforward tables and before transmission to the Digital-to-Analog Converters (DACs). 

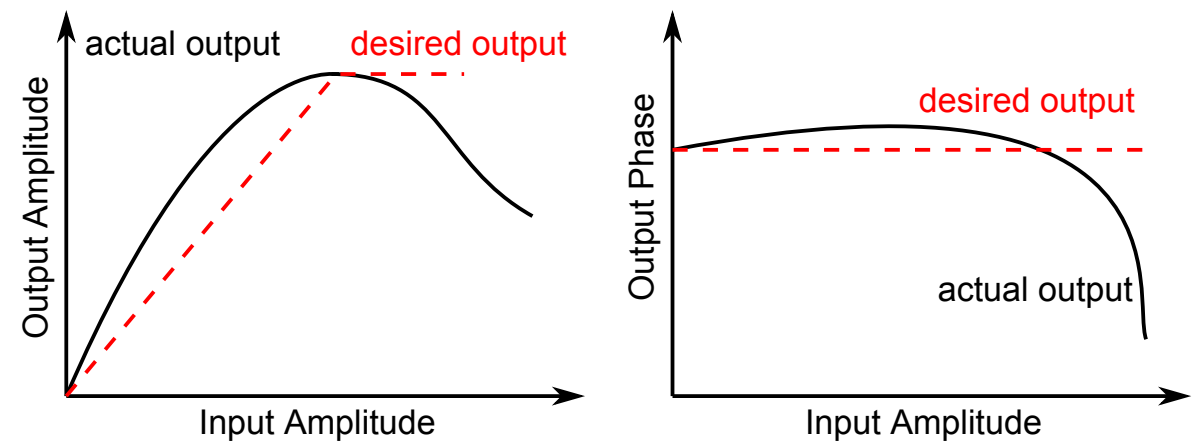

Figure 1: Schematic of the klystron amplitude and phase output characteristics.

Linearization concepts were already implemented in the past using analog circuits. The over the recent years improved capabilities of FPGAs allowed not only the implementation of digital LLRF feedback controllers but also the implementation of klystron linearization algorithms with high effectiveness and flexibility. Four algorithms implemented on FPGAs of two different manufacturers are described and compared in the following.

\section{Klystron Linearization Implemented at DESY}

At DESY the Free Electron Laser in Hamburg (FLASH) $[3$ is operated, which uses the same superconducting TESLA-type 9-cell cavity technology [4] as will be used in ILC. The digital LLRF control system at FLASH was based on the VME standard, before it was updated to uTCA.4 [5]. For the VME system a predistortion-type squared amplitude dependent direct lookup table

40 (LUT)-based klystron linearization was implemented [6, 7, which allowed a complex correction corresponding to

$$
\left(\begin{array}{l}
I^{\prime} \\
Q^{\prime}
\end{array}\right)=\left(\begin{array}{cc}
f_{i}\left(A^{2}\right) & -f_{q}\left(A^{2}\right) \\
f_{q}\left(A^{2}\right) & f_{i}\left(A^{2}\right)
\end{array}\right)\left(\begin{array}{l}
I \\
Q
\end{array}\right),
$$

where $I$ and $Q$ is the input of the linearization algorithm, $f_{i}\left(A^{2}\right)$ and $f_{q}\left(A^{2}\right)$ are the correction factors, and $I^{\prime}$ and $Q^{\prime}$ is the output of the algorithm. 
As part of the presented study this concept was reintroduced for the uTCA.4-

45 algorithm was a Xilinx Kintex 7 (K355/K420) FPGA on the LLRF controller card (uTC). The firmware creation tool set covered Notepad++, ISE Design Suite 14.4, and ISim. The schematic of the implemented VHDL package is shown in Figure 2 ,

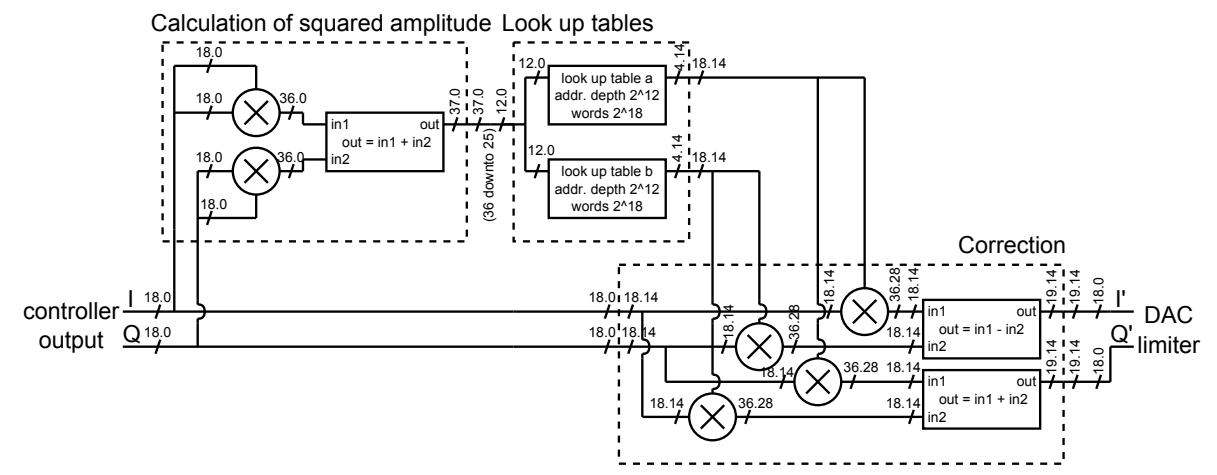

Figure 2: Schematic of the direct lookup table-based klystron linearization package.

From the 18-bit $I$ and $Q$ input signals the squared amplitude is computed using two multipliers and one adder. The resulting 37-bit word is truncated to 12 bits and used as the address of the lookup tables. The 16-bit output words of both lookup tables are applied to input $I$ and $Q$ values corresponding to a complex multiplication using four multipliers, one adder, and one subtracter.

${ }_{55}$ The resulting signals are truncated to 18-bit signals called $I^{\prime}$ and $Q^{\prime}$ and are outputted.

One unique feature of the implementation presented is the compatibility with the FPGAs on the uTCA.4-based hardware used at DESY. This means the klystron linearization package can be implemented and used not only in the digital LLRF control system at FLASH but also at the upcoming European X-Ray Free Electron Laser (European X-FEL) [9]. A second unique feature of the presented package is an improved MATLAB script for the generation of the lookup tables, which delivers more accurate results especially at low input amplitudes. 


\section{Klystron Linearization Implemented at FNAL}

At FNAL ASTA [10] is under construction. Since it is beside an user machine also an ILC R\&D accelerator, the digital LLRF control system was designed with ILC in mind.

As part of the presented study three kinds of predistortion-type amplitude dependent klystron linearization algorithms were implemented and tested. The target hardware was an Altera Cyclone II FPGA on the multi-cavity field control (MFC) module [11. The tools used for the manipulation and creation of the FPGA firmware were Matlab 2012b, Simulink 2012b, DSP Builder 13.0, ModelSim, and Quartus II 13.0. The first algorithm implemented was designed 75 for the linearization of the amplitude only [8]. Its principle is based on equation (2).

$$
\left(\begin{array}{c}
I_{\text {out }} \\
Q_{\text {out }}
\end{array}\right)=f_{\text {corr }}(A)\left(\begin{array}{c}
I_{\text {in }} \\
Q_{\text {in }}
\end{array}\right)
$$

Figure 3 shows a schematic of the linearization algorithm implemented. From the $I_{i n}$ and $Q_{i n}$ input values the amplitude $A$ is computed by $A=\sqrt{I_{i n}^{2}+Q_{i n}^{2}}$. For the implementation of the square root function a proprietary block of the Altera DSP Builder library was used. By a 3rd order polynomial function depending on the amplitude $A$ a correction factor $f_{\text {corr }}$ is calculated. Furthermore an amplitude limitation is included, which compares the expected output amplitude to a preset limit. If the limit is exceeded the correction factor is set to $f_{\text {corr }}=\frac{\text { limit }}{A}$. If the limit is not exceeded the correction factor is not changed and applied to the $I_{i n}$ and $Q_{i n}$ input values. By two switches the linearized or the original $I_{\text {in }}$ and $Q_{i n}$ values can be chosen as the output $I_{\text {out }}$ and $Q_{\text {out }}$.

The second implemented linearization algorithm was an upgrade of the previous one, which added the support of phase linearization. It is based on the equation (3)

$$
\left(\begin{array}{c}
I_{\text {out }} \\
Q_{\text {out }}
\end{array}\right)=\left(\begin{array}{cc}
f_{i}(A) & -f_{q}(A) \\
f_{q}(A) & f_{i}(A)
\end{array}\right)\left(\begin{array}{c}
I_{\text {in }} \\
Q_{\text {in }}
\end{array}\right),
$$




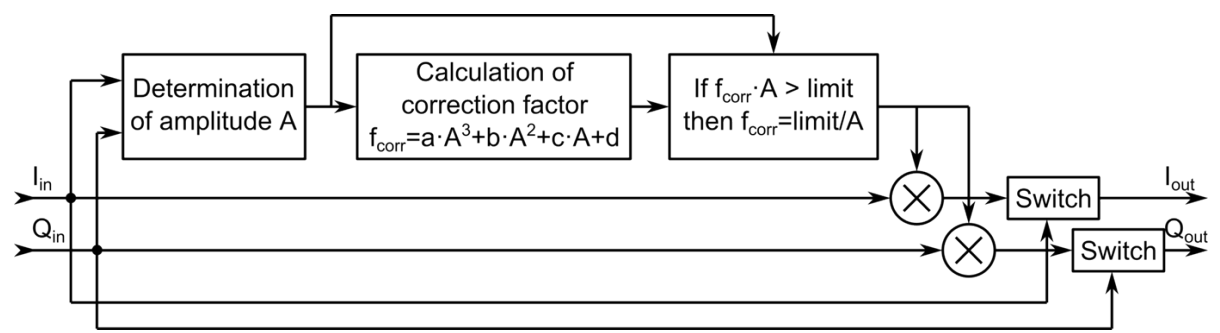

Figure 3: Schematic of third order polynomal-based klystron linearization algorithm.

where $f_{i}(A)$ and $f_{q}(A)$ are 2nd order polynomial functions. In Figure 4 the schematic of the implemented algorithm is shown. From the $I_{\text {in }}$ and $Q_{i n}$ input values the amplitude is computed. Based on this $I$ and $Q$ correction factors are calculated by two 2nd order polynomial functions. The computed $I$ and $Q$ correction factors are applied to the input $I_{i n}$ and $Q_{i n}$ values using a complex multiplication realized by four multipliers, one subtracter, and one adder. From the corrected $I_{i n}$ and $Q_{i n}$ values the amplitude is computed and compared to a predefined limit. If the amplitude exceeds the limit, a correction factor $f_{\text {corr }, l}=\frac{\text { limit }}{B}$ is applied. If the limit is not exceeded, the the correction factor $f_{c o r r, l}=1$ is applied, leaving the corrected $I_{i n}$ and $Q_{i n}$ unchanged. By two switches the linearized and amplitude limited or the original $I_{i n}$ and $Q_{i n}$ values can be chosen as the output $I_{\text {out }}$ and $Q_{\text {out }}$.

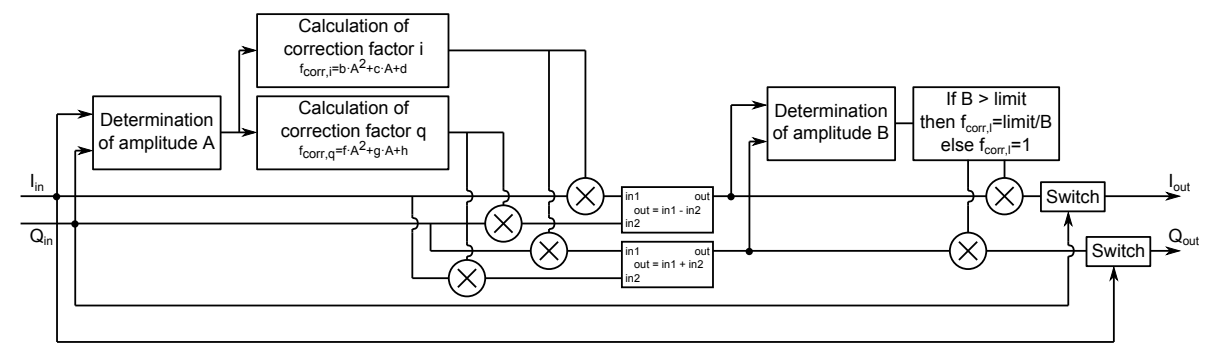

Figure 4: Schematic of second order polynomal-based klystron linearization algorithm.

In the case of the third implemented linearization algorithm the correction is also based on equation (3), but in this case $f_{i}(A)$ and $f_{q}(A)$ are created by lookup tables with interpolation [12]. The lookup table with interpolation-based algo- 
first lookup table the nodes $y_{n}$ are stored, similar to the case of a direct lookup table. In the second lookup table, the slopes between the nodes $m_{n}$ calculated by the differential quotient are stored. The output value of the algorithm $y$ is calculated by

$$
y=y_{n}+\Delta x \cdot m_{n}
$$

where $\Delta x=x-x_{n} . \quad x$ is the input value $x$ and $x_{n}$ the to the node $y_{n}$ corresponding $\mathrm{x}$ value. Figure 5 shows a schematic of the implementation of the klystron linearization algorithm. Since a complex correction factor is generated, the lookup table with interpolation algorithm is implemented two times. Beside the change of the method of the generation of the complex correction factor, the signal flow is the same as described previously for the klystron linearization based on two 2 nd order polynomial functions.

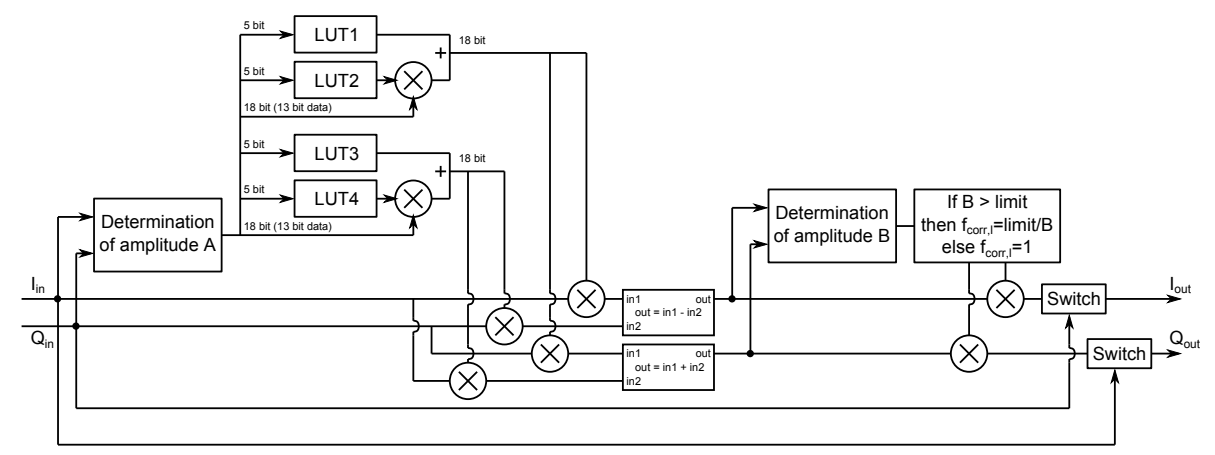

Figure 5: Schematic of the lookup table with interpolation-based klystron linearization package.

\section{Comparison of Algorithms}

In Table 1 the clock cycles required for the execution of the linearization algorithms and for the execution of the amplitude limitations are compared. 
putations are performed in a parallel process. The loop delays added due to the application of the linearization algorithms are also listed in Table 1.

Table 1: Comparison of delays. (* including amplitude limiter)

\begin{tabular}{lccc}
\hline Algorithm & $\begin{array}{c}\text { Lin. } \\
\text { [clk. cyc.] }\end{array}$ & $\begin{array}{c}\text { Limiter } \\
\text { [clk. cyc. }\end{array}$ & $\begin{array}{c}\text { Add. loop del. } \\
\text { [clk. cyc. }\end{array}$ \\
\hline Direct LUT & 6 & - & 2 \\
3rd order & $31^{*}$ & - & 1 \\
2nd order & 20 & 19 & 3 \\
LUT w/int. & 19 & 19 & 3 \\
\hline
\end{tabular}

The computation times of the linearization and amplitude limitation algorithms in the case of the 3rd order polynomial function-based, the 2nd order polynomial function-based, and lookup table with interpolation-based algorithms are dominated by the computation of the amplitude using the square root block, which is pipelined with 12 clocks. The algorithm with the shortest computation time is the one based on direct lookup tables. If the application allows quantization errors this is the method of choice. The second fasted algorithm is the one based on the lookup tables with interpolation.

From the viewpoint of total added loop delay, the 3rd order polynomial function-based algorithm with only 1 clock cycle added is the best. This is due to the fact, that in the linearization algorithm already the amplitude limitation is included. This algorithm is only applicable, when solely the amplitude has to be linearized. All other three algorithms add 3 clock cycles of loop delay. This is also true for the case the direct lookup table-based algorithm is combined with an amplitude limiter. Furthermore is should be kept in mind that the algorithms are implemented on different FPGAs with different clock frequencies. The Xilinx Kintex 7 on which the direct lookup table algorithm was implemented is clocked at a frequency of $81.25 \mathrm{MHz}$. The Altera Cyclone II on which all remaining algorithms were implemented are clocked at a frequency of $62.5 \mathrm{MHz}$.

Table 2 compares the resources used on the Cyclone II FPGA in the cases 
of the different linearization algorithms. This shows that regarding the usage of total logic elements the 3rd order polynomial function-based and the lookup table with interpolation-based algorithms are most resource efficient. Regarding the hardware multipliers the lookup table with interpolation-based algorithm is most efficient.

Table 2: FPGA resource usage.

\begin{tabular}{lcc}
\hline & $\begin{array}{c}\text { Total } \\
\text { logic } \\
\text { elements }\end{array}$ & $\begin{array}{c}9 \text { bit } \\
\text { hardware } \\
\text { multipliers }\end{array}$ \\
\hline w/o lin. & $28 \%$ & $76 \%$ \\
3rd order & $31 \%$ & $99 \%$ \\
2nd order & $32 \%$ & $91 \%$ \\
LUT w/int. & $31 \%$ & $84 \%$ \\
\hline
\end{tabular}

Beside the comparison of the hardware utilization and performance also the expected linearization performance was compared. To this end an arbitrary correction function was generated. This function is shown in Figure 6 in green. Furthermore this function was modeled using all algorithms mentioned above. The results are also shown in Figure 6. Beside this also an algorithm based on a lookup table with interpolation and an adaptive node distribution, which is intended to be implemented in the future, is included.

155 In order to compare the expected modeling performances of the algorithms the differences between the original function and the modeled output was computed. The result is shown in Figure 7.

From the deviation plots the sums of absolute values of the deviations over all plotted points were computed for all simulated algorithms. This yields a similar information as the integral over the error functions. The computed sums are listed in Table 3

This shows from the viewpoint of expected linearization performance in theory the best choice within the group of implemented algorithms is the lookup 


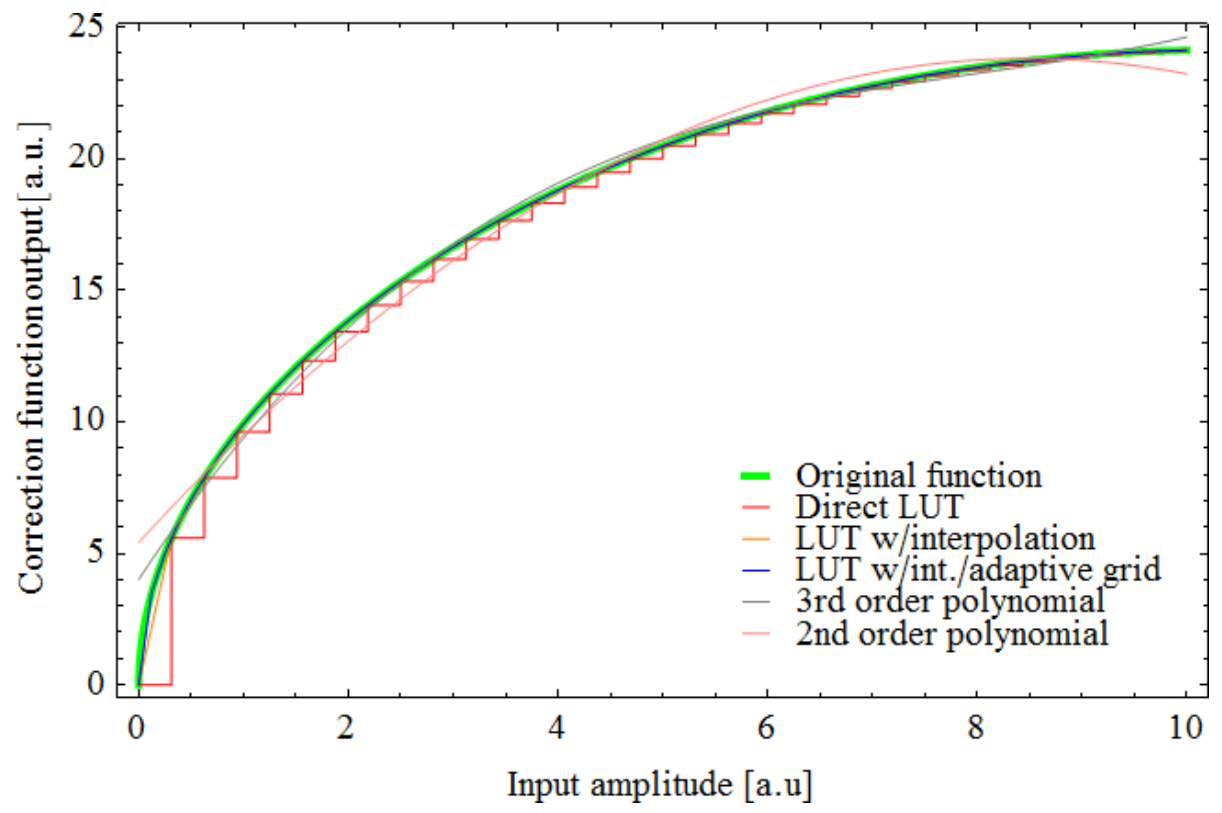

Figure 6: Simulated arbitrary correction function output [a.u.] versus input amplitude [a.u.] for the original function (green), a direct LUT (red), LUT with interpolation (orange), LUT with interpolation and an adaptive grid (blue), a 3rd order polynomial function-based algorithm (grey), and a 2nd order polynomial function-based (pink).

table with interpolation-based klystron linearization. Due to the interpolation the quantization errors are eliminated. Even it is notable that also the 3rd order polynomial function based algorithm yields a better performance than the direct lookup table solution, it should be kept in mind, that the performance strongly depends on the lookup table grid spacing. The best performance of all algorithms presented in the test yielded the lookup table with interpolation and adaptive grid spacing-based algorithm. It is intended to implement and test it in future studies.

\section{Test of Algorithms}

The functionality of the direct lookup table-based linearization algorithm implemented at DESY could only be verified in a iSim simulation. To this 


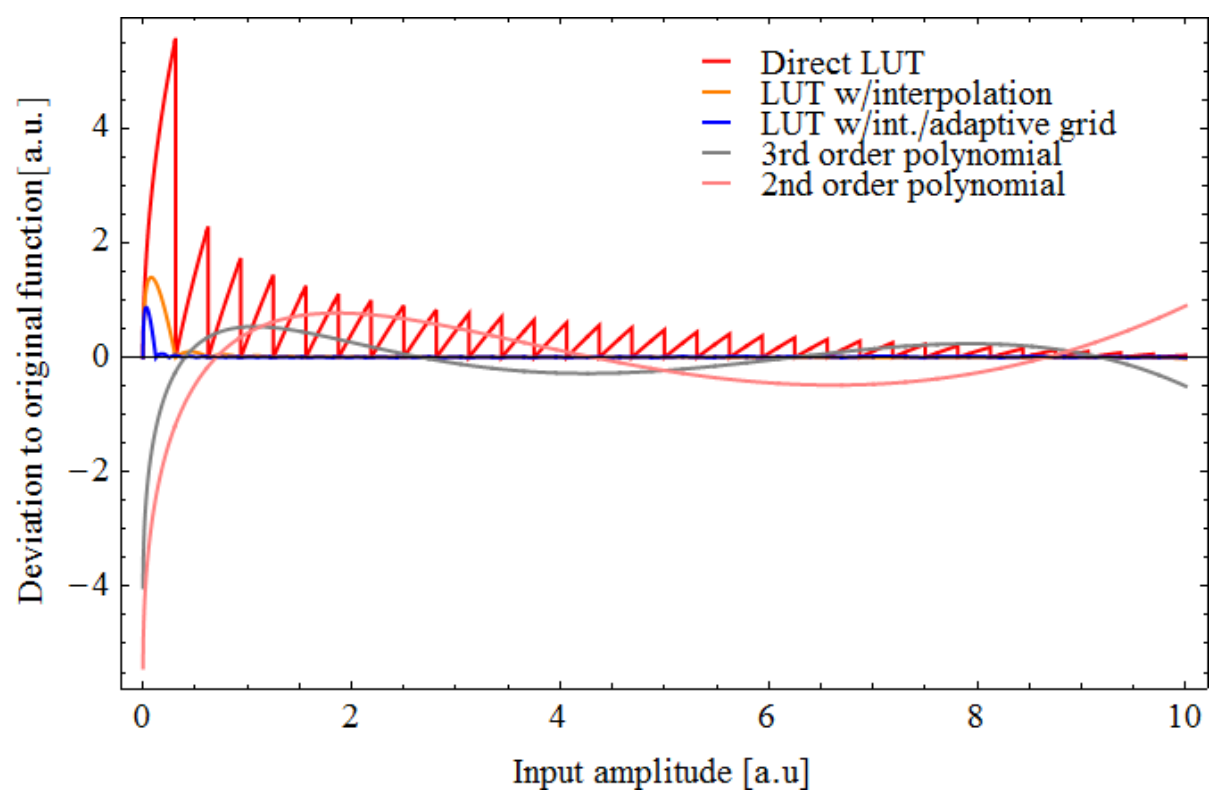

Figure 7: Simulated deviation to original arbitrary correction function [a.u.] versus input amplitude [a.u.] for a direct LUT (red), LUT with interpolation (orange), LUT with interpolation and an adaptive grid (blue), a 3rd order polynomial function-based algorithm (grey), and a 2nd order polynomial function-based (pink).

end a non-linear klystron characteristic in amplitude and phase recorded at the $10 \mathrm{MW}$ klystron of FLASH (ACC67) as shown in Figure 8 in blue was implemented in a VHDL test bench. Furthermore a MATLAB script for the calculation of the lookup table content was written. The result of the simulation of the linearization is shown in Figure 8 in purple. The amplitude was linearized sufficiently. The output phase stayed constant except a slight fluctuation at high input amplitudes. With this a proof of concept was demonstrated.

The three linearization algorithms implemented at FNAL were tested at ASTA in two different setups. In preparation for the test of the klystron linearization algorithm a klystron characterization was conducted in all cases. To this end under open loop operation of the klystron a feedforward (FF) amplitude scan over the whole range possible from 0 to 1 [a.u.] was performed. The klystron output characteristics in terms of amplitude, square root of the output 
Table 3: Sums of absolute values of deviations over all plotted points for all simulated algorithms.

\begin{tabular}{lc}
\hline Algorithm & Sum of errors \\
\hline Direct LUT & 6752.3 \\
LUT w/int. & 588.1 \\
LUT w/int./ad. grid & 250.8 \\
3rd order & 3964.5 \\
2nd order & 7584.0 \\
\hline
\end{tabular}

power, and phase were recoded. Based on this the parameters and lookup tables for the three linearization algorithms were computed.

The first algorithm tested was the 3rd order polynomial function-based linearization. In the corresponding test setup the klystron was directly run into a dummy load. Since this linearization algorithm was designed for amplitude linearization only, no phase information is discussed in this case. Figure 9 shows the klystron output amplitude $[\operatorname{sqrt}(\mathrm{kW})]$ versus the $\mathrm{FF}$ amplitude during the characterization, which means without the linearization, in blue. In the same figure the red plot shows the klystron output amplitude characteristic with the linearization activated. The achieved output agrees very well with in average less than $1 \%$ deviation from the simulated linearized output shown in Figure 9 in green. With this a successful implementation of the 3rd order polynomial function-based linearization algorithm was demonstrated.

At the time of the test run of the linearization algorithms based on the 2nd order polynomial functions and the lookup table with interpolation, the $5 \mathrm{MW}$ klystron was connected to cavity 5 of the ILC RF unit. Since the test run was conducted during cavity conditioning, the administrative limit for the klystron output power was $800 \mathrm{~kW}$. In order to drive the klystron into saturation at such a low output power, the attenuation on the RF drive was reduced to $2 \mathrm{~dB}$. Furthermore the klystron gun voltage was reduced to $-89.6 \mathrm{kV}$. Figure 10 shows the klystron output amplitude versus the FF amplitude during characterization 

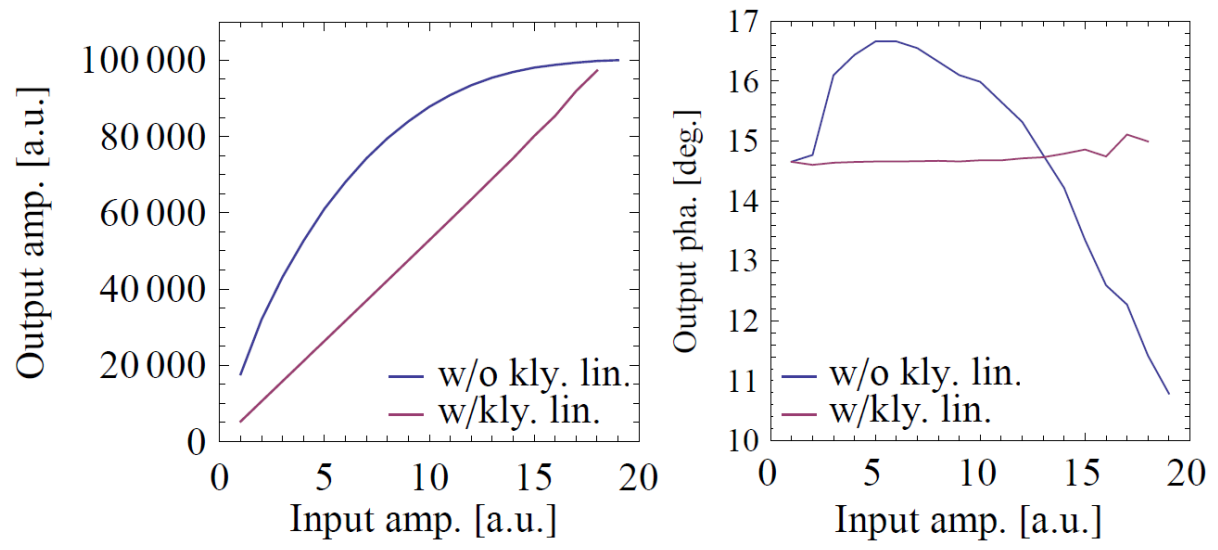

Figure 8: Plots of result of iSim simulation without the klystron linearization (blue) and with linearization (purple). Left: Klystron linearization algorithm output amplitude [a.u.] versus input amplitude [a.u.]. Right: Klystron linearization algorithm output phase [0] versus input amplitude [a.u.].

and in the case of no linearization active in yellow. The same figure shows also the klystron output amplitudes in case the two linearization algorithms were active (blue for the 2nd order polynomial-based and purple for the lookup table with interpolation-based). The target amplitude characteristic is plotted in green. In order to analyze the linearization performances in more detail Figure 11 shows the differences between the target and achieved klystron output amplitudes $[\operatorname{sqrt}(\mathrm{kW})]$ versus $\mathrm{FF}$ amplitude [a.u.] for 2nd order polynomial function-based linearization in blue and for the LUT with interpolation-based linearization purple. From this it can be seen that the 2nd order polynomial function-based klystron linearization has the better linearization performance concerning the amplitude.

Figure 12 shows the klystron output phase for the case no linearization was active in yellow as well as the target phase characteristic in green. In the same figure the also the phases during the 2nd order polynomial function-based (blue) and the lookup table with interpolation-based (purple) algorithms were active are shown. In Table 4 the maximal phase rotations for all cases are summarized. 


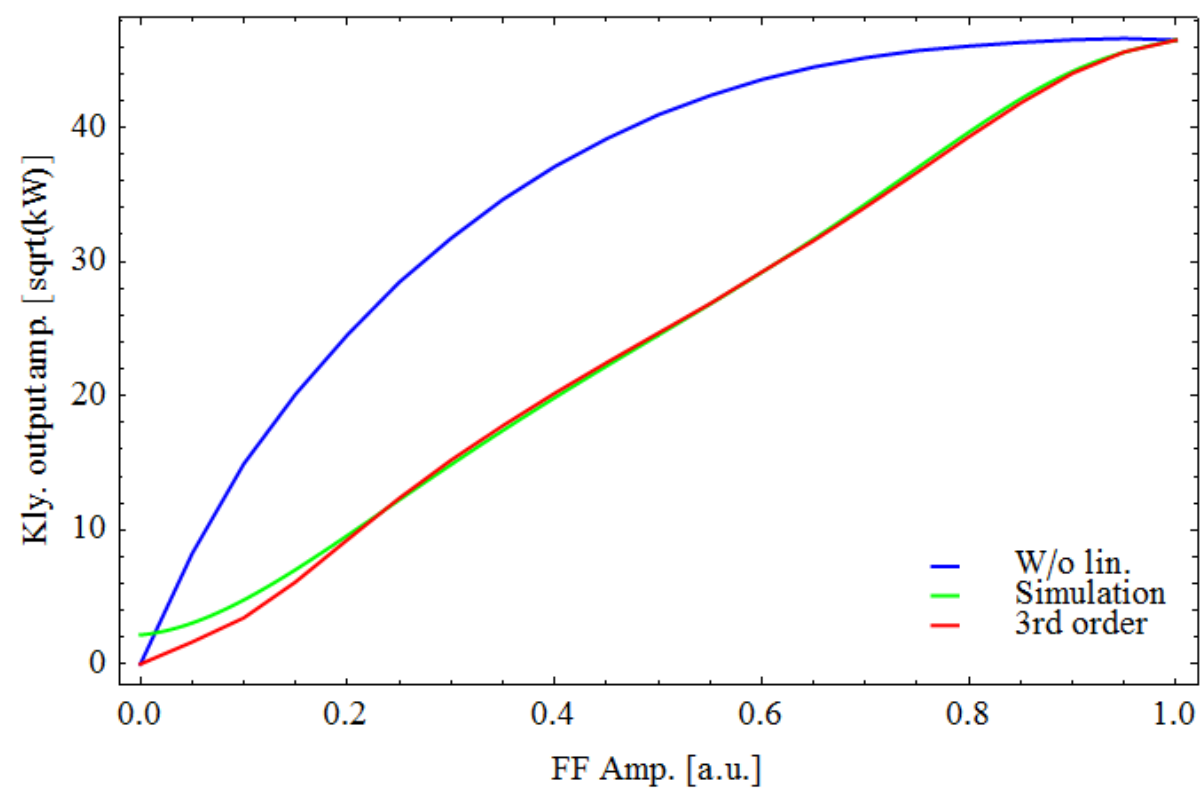

Figure 9: Klystron output amplitude [sqrt $(k W)]$ versus FF amplitude [a.u.] of test of 3rd order polynomial function-based linearization: without linearization (blue), simulated linearized output (green), with 3rd order polynomial function-based linearization (red).

It can be seen that the lookup table with interpolation-based klystron linearization algorithm has the best linearization performance concerning the phase with $13^{\circ}$ over the whole $\mathrm{FF}$ amplitude range. For the $\mathrm{FF}$ amplitude range until the point of saturation the phase rotation is with only $5^{\circ}$ even lower.

Table 4: FPGA resource usage.

\begin{tabular}{lc}
\hline & $\begin{array}{c}\text { Phase rotation over } \\
\text { FF amplitude range }\end{array}$ \\
\hline w/o lin. & $40^{\circ}$ \\
2nd order & $15^{\circ}$ \\
LUT w/int. & $13^{\circ}$ \\
\hline
\end{tabular}

The fluctuations in amplitude and phase in the case of the lookup table with interpolation-based algorithm originate from an error regarding the computa- 


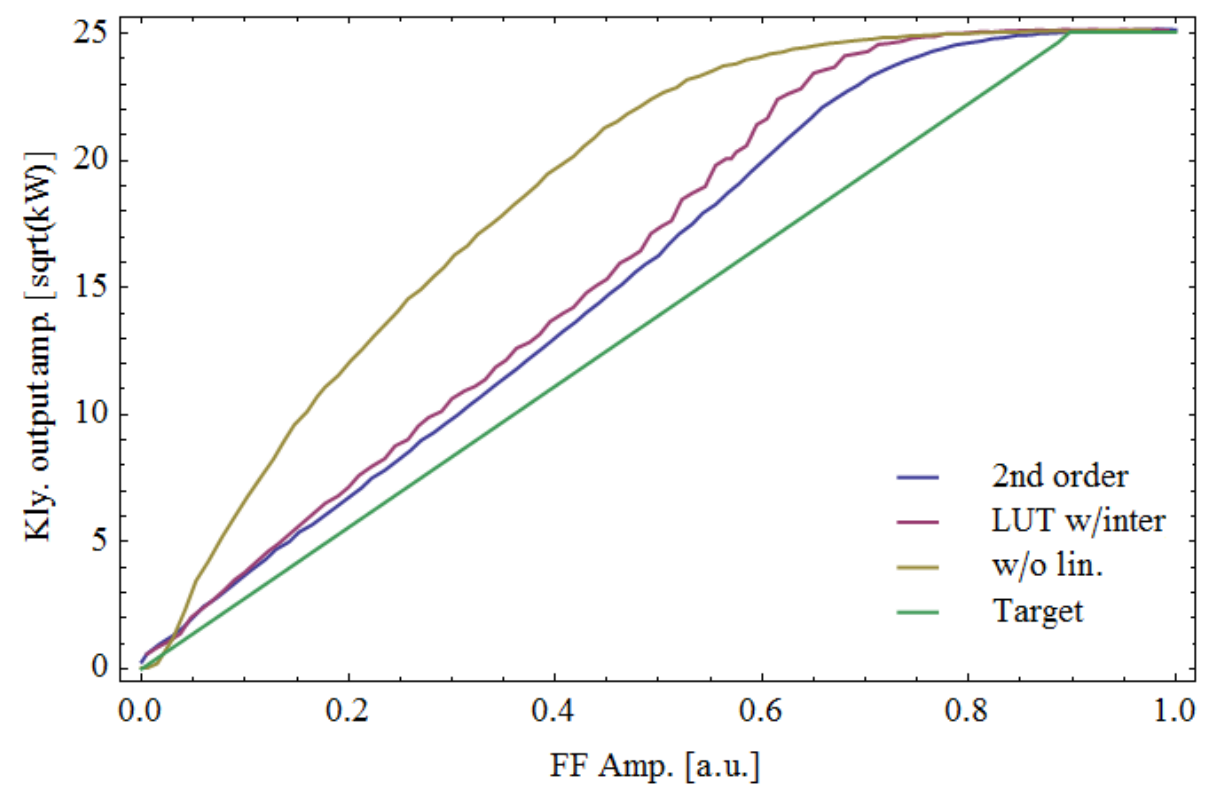

Figure 10: Klystron output amplitude [sqrt $(k W)]$ versus FF amplitude [a.u.]: 2nd order polynomial function-based linearization (blue), and with LUT with interpolation-based linearization (purple), without linearization (yellow), target amplitude (green).

tion of the slope lookup tables. Nevertheless with the presented data a prove of concept of both the 2nd order polynomial function-based and the lookup table with interpolation-based linearization algorithms was demonstrated.

\section{Cavity-Klystron Simulator}

Nowadays FPGA-based cavity simulators are a common tool for the development and test of digital LLRF control system in times no actual cavity is available. Nevertheless, simulating only a cavity does not reflect the entire actual system including high power amplifiers such as e.g. klystrons. The situation can be improved by including an active analog amplifier within the feedback loop simulating non-linear characteristics such as a saturation behavior. The disadvantage of using such an analog amplifier is that it has a different input-to-output characteristic (especially regarding the phase) compared to a 


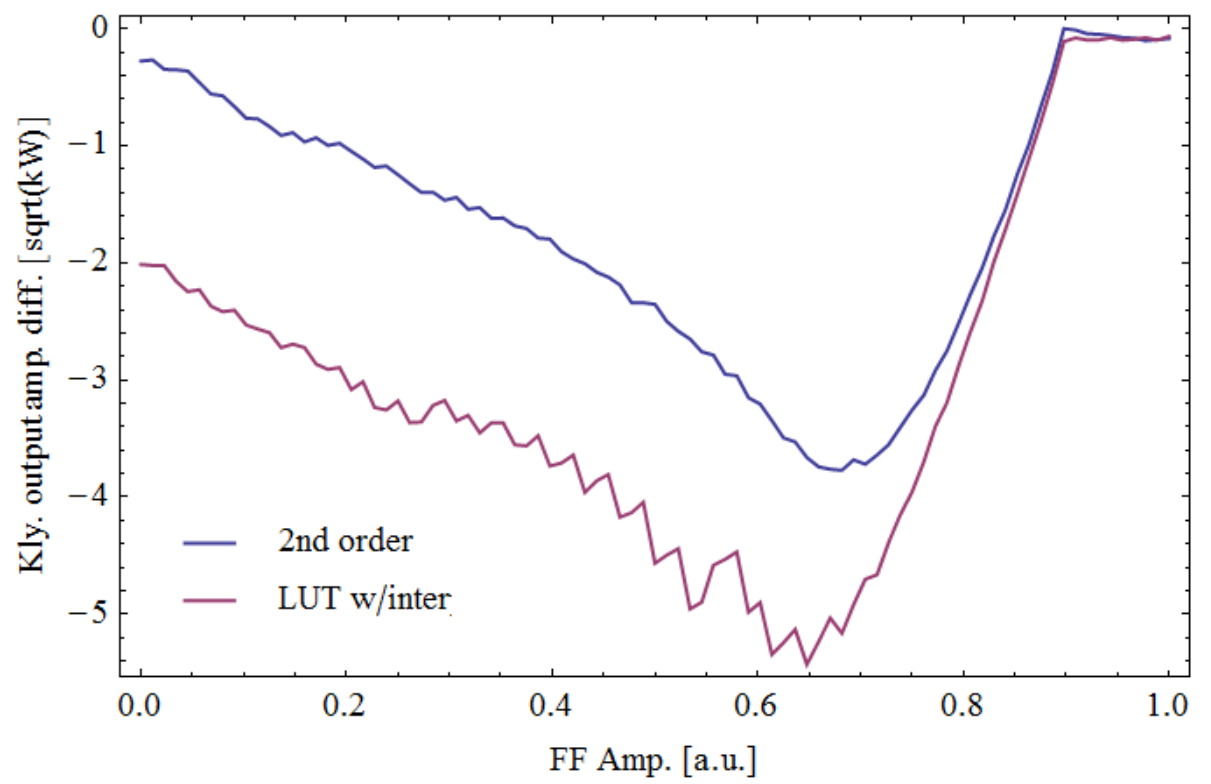

Figure 11: Differences between target and achieved klystron output amplitude [sqrt $(k W)]$ versus FF amplitude [a.u.]: 2nd order polynomial function-based linearization (blue), and with LUT with interpolation-based linearization (purple).

klystron. This situation can be overcome by extending the FPGA-based cavity simulator by a klystron simulator package. By this a realistic test environment for offline developments and tests can be realized. Since such an FPGA-based klystron simulator is highly configurable and flexible, it is possible to simulate any possible non-linear characteristics of the klystron and other components both in amplitude and phase.

A klystron-cavity simulator was developed and implemented on a uTCA AMC board at KEK. It consists mainly of two packages, the first for the klystron simulator and the second for the cavity simulator. The klystron simulator package is realized using a direct lookup table-based algorithm, similar to the one implemented for the klystron linearization at DESY (see above). The cavity 


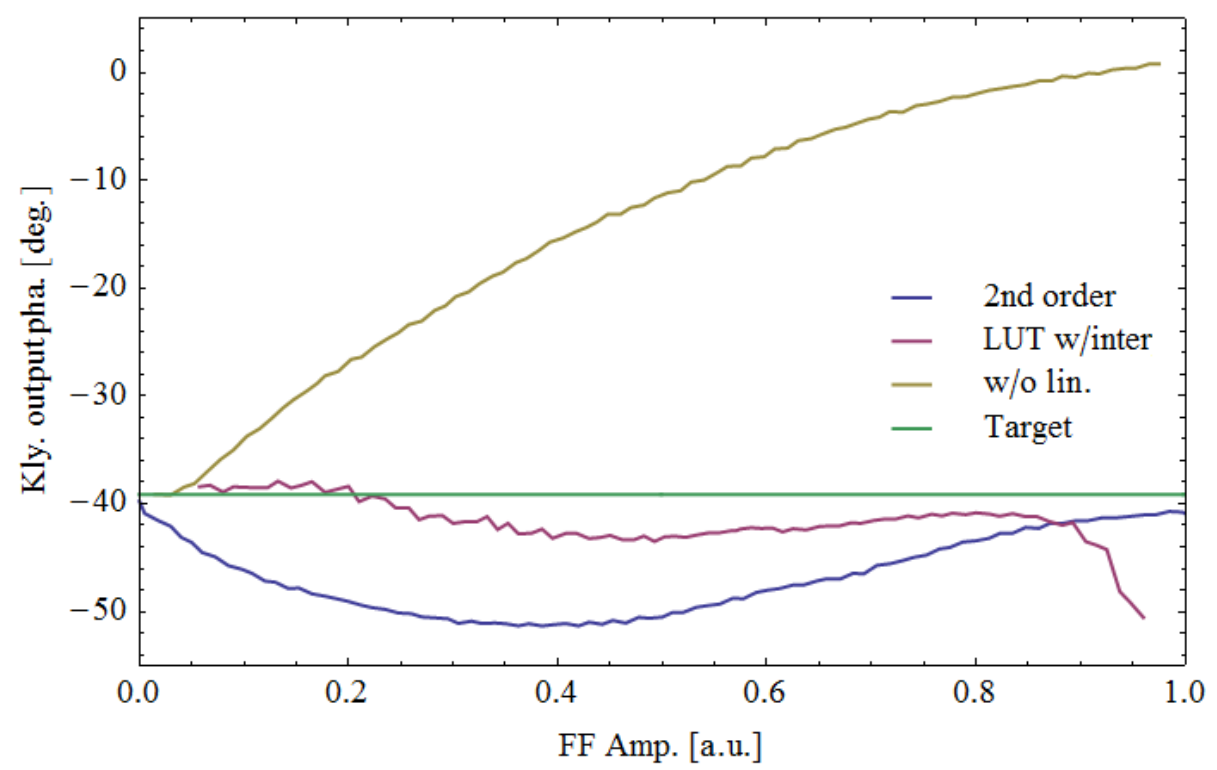

Figure 12: Klystron output phase [ ${ }^{0}$ ] versus FF amplitude [a.u.]: 2nd order polynomial function-based linearization (blue), and with LUT with interpolation-based linearization (purple), without linearization (yellow), target phase (green).

$$
\begin{array}{r}
{\left[\begin{array}{c}
V_{i, n} \\
V_{q, n}
\end{array}\right]=\left[\begin{array}{cc}
1-T \omega_{1 / 2} & -\Delta \omega \\
T \Delta \omega & 1-T \omega_{1 / 2}
\end{array}\right]\left[\begin{array}{c}
V_{i, n-1} \\
V_{q, n-1}
\end{array}\right]} \\
+T \omega_{1 / 2} R_{L}\left[\begin{array}{c}
I_{i, n-1} \\
I_{q, n-1}
\end{array}\right] .
\end{array}
$$

For the implementation the algorithm was simplified by assuming the case of no detuning. Figure 13 shows a simplified schematic of the entire klystroncavity algorithm implementation on the uTCA AMC board.

The VHDL code for the klystron-cavity simulator was written in Notepad $260++$, compiled in ISE, and tested in the ISim simulator. After sufficient testing the klystron-cavity simulator algorithm was programmed to the FPGA on a uTCA AMC board. This board was connected with a second uTCA AMC 


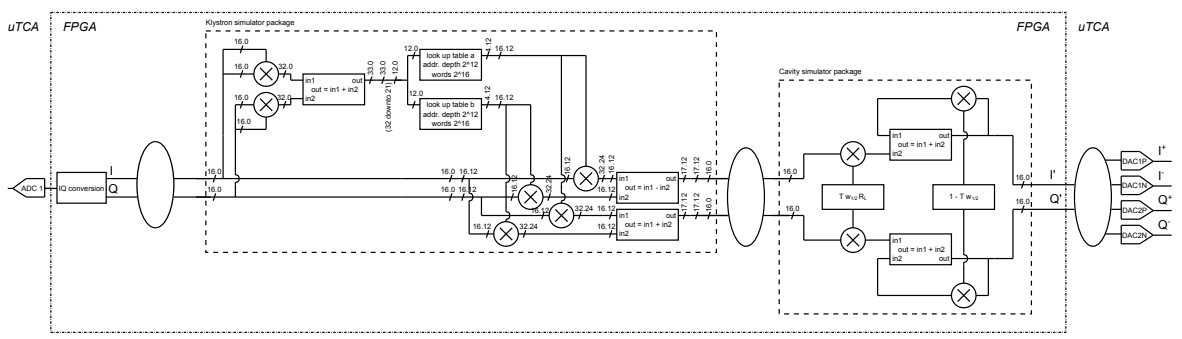

Figure 13: Simplified schematic of the klystron-cavity algorithm implementation on the uTCA AMC board (FB7).

board, on which the original LLRF controller firmware was kept as described in the following and shown in Figure 14 . The features of the LLRF controller algorithm important for the test setup are a CAV SIM IIR filter, a proportional and integral gain, and the addition of FF tables. The output of the DACs of controller board are converted from differential to single end using two analog converters. The restored I and $\mathrm{Q}$ values are sent to a IQ vector modulator. A second input of the vector modulator is a $10 \mathrm{MHz}$ signal. By the vector modulator the I and Q values are converted from a baseband to an IF signal. This signal is split into two paths. One is connected to the ADC2 channel of the controller board. The second one is connected to the ADC1 channel of the klystron-cavity simulator board. Its differential baseband I and Q output is converted to single end signals by two analog converters. The single end I and $\mathrm{Q}$ signals are fed to a IQ vector modulator. Beside this also a $10 \mathrm{MHz}$ signal is fed to the IQ vector modulator. By this an IF signal is generated, which is filtered by a band pass filter and fed to ADC1 on the controller board.

With this configuration an open loop (FF only) as well as a closed loop (FB with or without FF) operation driving the klystron-cavity simulator was successfully demonstrated.

In a first test the functionality of the cavity simulator package was checked. To this end the response of the klystron-cavity simulator in an open loop (FF only) operation was observed. In order to engage in operation a previously recorded klystron characteristic was programmed to the lookup tables of the 


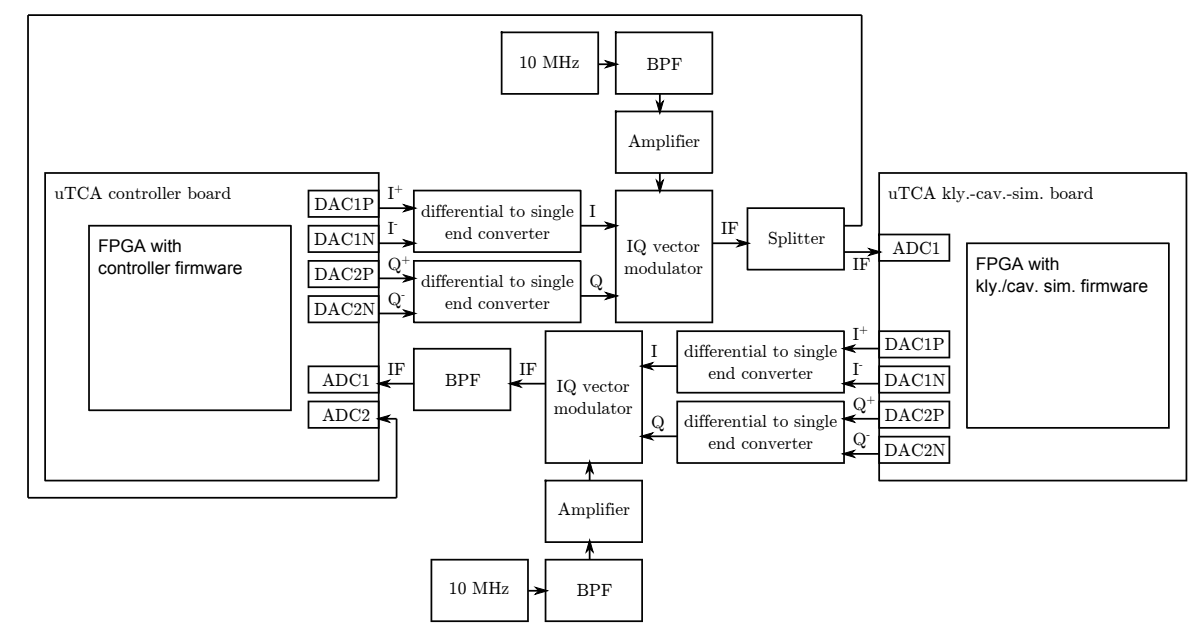

Figure 14: Simplified schematic of the test setup covering the klystron-cavity simulator board and the LLRF controller board.

klystron simulator package. The half bandwidth for the cavity simulator was set to $f_{1 / 2}=216.7 \mathrm{~Hz}$ corresponding a loaded $\mathrm{Q}$ value of $3 \cdot 10^{6}$. Figure 15 shows on top the output of the LLRF controller board, which is a rectangular pulse in amplitude and a constant phase. The same figure shows on the bottom the input of the LLRF controller board, which corresponds to the output of the klystron-cavity simulator. A typical cavity response can be observed.

In a second test the functionality of the klystron simulator package was checked. To this end a FF table amplitude scan was performed from 1000 to 6500 counts, while the DAC output of the klystron-cavity simulator was observed as a digital signal via EPICS. Figure 16 shows the output amplitude [counts] during the steady state condition versus the FF amplitude [counts] for the expected output in green and the measured DAC channel output of the simulator in red. The expected output plot is based on a simulation using the same lookup table content as programmed to the simulator board. The expected output and the measured output agree very well with deviations of less than

$2.5 \%$ at $\mathrm{FF}$ values below 1500 and above 5000 .

With these two test runs a proof of concept of the algorithm implementation 
DAC: $128.9137 \% \mathrm{rms}$

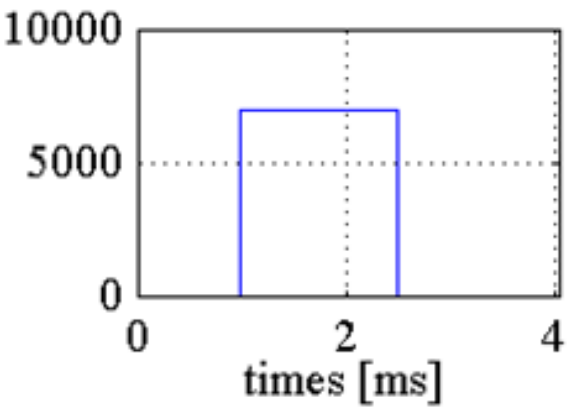

ADC1:113.8004\%rms

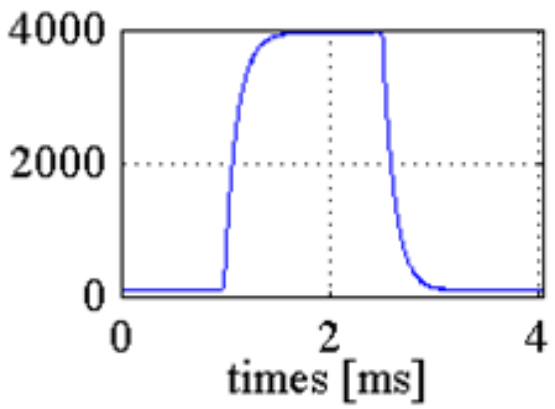

DAC:0deg.rms

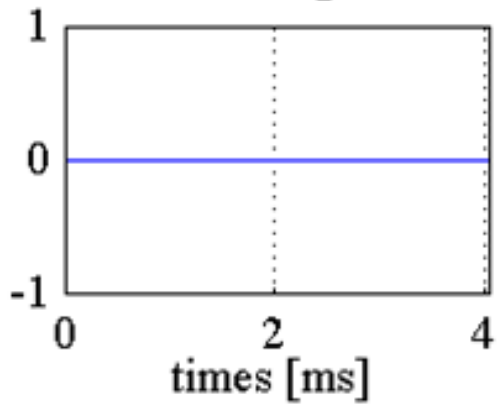

$\mathrm{ADC} 1: 35.9165$ deg.rms

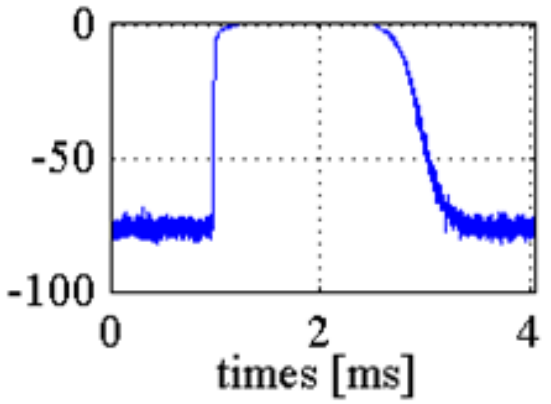

Figure 15: Top: DAC output amplitude [a.u.] (left) and phase [ ${ }^{\circ}$ ] (right) versus time [ms] of the LLRF controller. Bottom: ADC input amplitude [a.u.] (left) and phase [ ${ }^{\circ}$ ] (right) versus time [ms] of the LLRF controller.

was demonstrated.

\section{Conclusions}

In an international collaboration with DESY and FNAL total four FPGAbased predistortion-type klystron linearization algorithms were implemented and tested. At DESY a direct lookup table-based algorithm was implemented in the scope of the new $\mu$ TCA.4 hardware used for LLRF control at FLASH. The implementation was tested successfully in an iSim simulation resulting in a prove of concept.

At FNAL linearization algorithms based on one 3rd order polynomial function, two 2nd order polynomial functions, and lookup tables with interpolation 


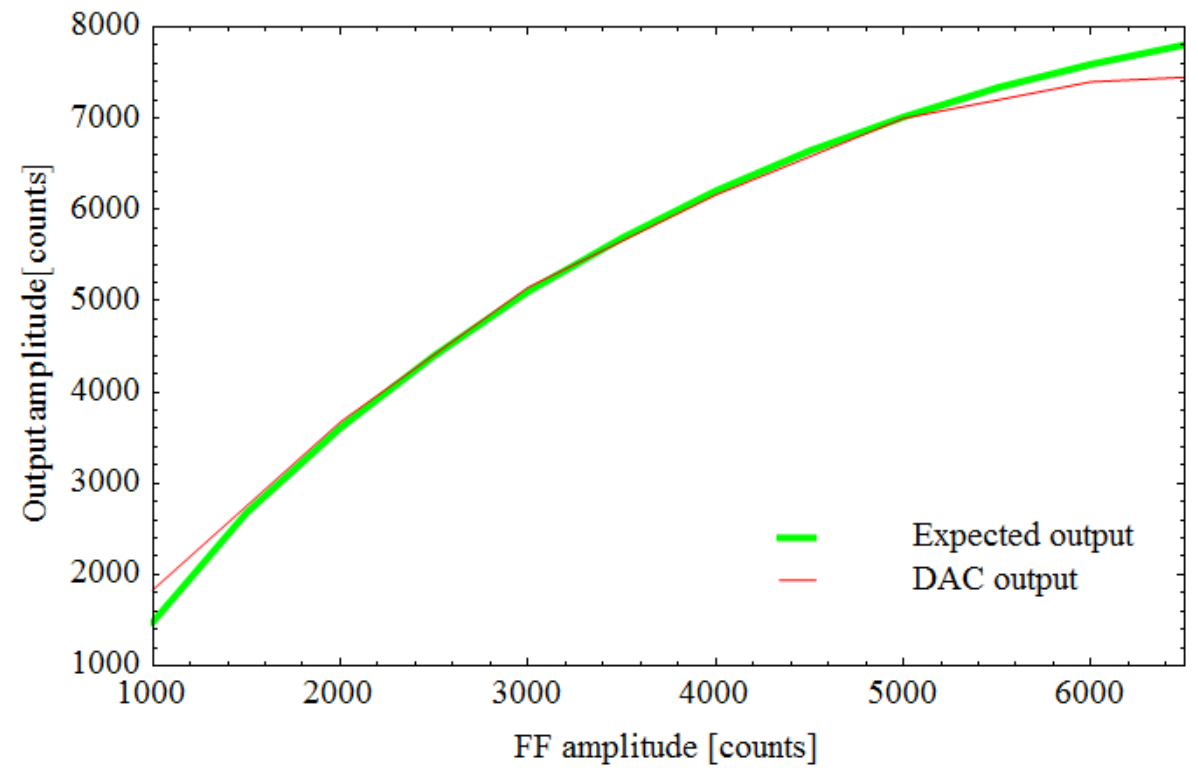

Figure 16: Output amplitude [counts] versus FF amplitude [counts]: for the expected output (green) and the measured output (red).

were implemented. In all cases the quantization of the output of the linearization algorithm was avoided. In the cases of the polynomial function-based algorithms furthermore the memory requirements could be reduced drastically, since only few configuration parameters had to be stored instead of lookup tables. The three algorithms were tested using a $5 \mathrm{MW}$ klystron at FNAL ASTA resulting in a successful implementation of the 3rd order polynomial-based algorithm and the prove of concept of the two other algorithms. Within the group of implemented and tested algorithms the one based on lookup tables with interpolation yields theoretically the best linearization performance.

It is proposed to add an adaptive adjustment of the spacing of the nodes to algorithm based on lookup tables with interpolation. In a simulation an improvement of modeling the correction function was demonstrated. It is intended to implement and test the improved algorithm in an actual setup.

The described linearization technique is not only suitable for ILC but is 
also for other accelerators and applications at which high efficiency RF usage is required.

Beside the development of the klystron linearization algorithms an FPGAbased klystron-cavity simulator was implemented and successfully tested in a development environment resulting in a prove of concept. The simulator provides a realistic environment for tests and developments of digital LLRF control systems while an actual klystron or cavity is not available.

\section{References}

[1] http://www.linearcollider.org

[2] "The International Linear Collider - Technical Design Report", CERN, FNAL, KEK (2013).

[3] R. Kammering, "Feedbacks and Automation at the Free Electron Laser in Hamburg (FLASH)", THPPC121, p. 1345, Proc. ICALEPCS2013, San Francisco (2013).

[4] "TESLA Technical Design Report - PART II - The Accelerator ", http://flash.desy.de

[5] J. Branlard, "Status Update of the uTCA LLRF System for XFEL and FLASH Upgrade" (Presentation), ILC 9mA Meeting (2011).

[6] W. Cichalewski, "Linearization of Microwave High Power Amplifiers Chain in the RF Systems of Linear Accelerators for FLASH and X-FEL" (PhD Thesis), Technical University of Lodz, Lodz (2008).

[7] P. Fafara, "FPGA-based Implementation of a Cavity Field Controller for FLASH and X-FEL", Meas. Sci. and Technol. 18 (2007) 2365-2371, (2007).

[8] M. Omet, "Development and Test of Klystron Linearization Packages for 350 FPGA-based Low Level RF Control Systems of ILC-like Electron Accelerators", Proc. RT2014, Nara (2014). 
[9] "The European X-Ray Free Electron Laser Technical Design Report", http://xfel.desy.de

[10] http://asta.fnal.gov/

355 [11] P. Varghese, "Multi-cavity Field Control (MFC) Module Description", FNAL, Batavia (2009).

[12] B. Chase, private communication, FNAL, Batavia (2014). 